\title{
Leifsonia aquatica: Case report and literature review
}

\author{
RICARDO VIANNA DE CARVALHO ${ }^{1}$, CÍNTIA SILVA SANTOS ${ }^{2}$, LOUISY SANCHES DOS SANTOS SANT'ANNA ${ }^{2}$, \\ FERNANDA FERREIRA LIMA ${ }^{1}$, RAPHAEL HIRATA JÚNIOR ${ }^{2}$, \\ MARÍLIA FOURNACIARI GRABOIS ${ }^{1}$ and ANA LUÍZA MATTOS-GUARALDI ${ }^{2}$
${ }^{1}$ Pediatric Oncology Surgery Department, National Cancer Center Institute Jose Alencar Gomes da Silva (INCA/RJ), Ministry of Health-Science and Technology, Rio de Janeiro 20230-130;
${ }^{2}$ Rio de Janeiro State University, (UERJ), Faculty of Medical Sciences, Discipline of Microbiology and Immunology, Laboratory of Diphtheria and Corynebacteria of Clinical Relevance, Rio de Janeiro 20551030, Brazil

Received October 26, 2018; Accepted August 17, 2021

DOI: $10.3892 / \mathrm{mco} .2021 .2387$

\begin{abstract}
Non-diphtheria Corynebacterium species have been increasingly recognized as multidrug resistant pathogens that also infect immunocompromised patients. Automated and semi-automated phenotypic tests have been used by clinical laboratories for detection of these gram-positive rods. The present case report describes the rare pediatric case of L. aquatica isolated in central venous catheter blood cultures during chemotherapy treatment for Wilms tumor and adds to the knowledge on this infection with regard to pediatric cancer. The clinical aspects of this patient and opportunities for improving treatment were reviewed. Additionally, a review of the literature revealed no other case report involving cancer and a pediatric patient with documented L. aquatica bacteremia. Corynebacterial infections are considered uncommon, but in recent decades' reports on infection with bacterium are increasing in frequency, particularly in nosocomial immunocompromised patients.
\end{abstract}

\section{Introduction}

Toxigenic Corynebacterium Diphtheriae is a major cause of morbidity and mortality in regions of the world where immunization against diphtheria is not universally available. Coryneform bacteria are a diverse group of aerobically growing non-spore forming, irregularly shaped grampositive rods. Geographical variations in the frequency of isolated species and variations in natural and acquired

Correspondence to: Dr Ricardo Vianna de Carvalho, Pediatric Oncology Surgery Department, National Cancer Center Institute Jose Alencar Gomes da Silva (INCA/RJ), Ministry of Health-Science and Technology, Praça da Cruz Vermelha 23, Rio de Janeiro 20230-130, Brazil

E-mail: ricardovianna@yahoo.com

Key words: corynebacterial, Leifsonia aquatica, pediatric cancer, infection antimicrobial resistance have been described (1). Diagnosis of coryneform bacteria remains a challenge for routine checks, due to the large number of different species belonging to this group $(2,3)$. Isolates of coryneform gram-positive rods are often difficult to identify for diagnostic laboratories. Modern technologies such as MALDI-TOF MS and/or RNA sequencing molecular detection methods for bacterial pathogens in fluids provide sensitive, specific and rapid results (4).

The incidence of Corynebacterium spp. as pathogens of nosocomial infections associated with septicemia, endocarditis, infections of surgical wounds and infections related to the use of invasive medical devices is increasing. Blood culture is considered the gold standard for diagnosis of bacteremia. The incidence and clinical presentations of Corynebacterium spp. infections differ in children and adults (5).

Non-diphtheria Corynebacterium species have been increasingly recognized as multidrug resistant (MDR) pathogens that also infect immunocompromised patients (5), and Vancomycin is still considered the primary drug of choice in the control of this infection. The indication of the removal of catheters is recommended in guidelines, such as the Clinical and Laboratory Standards Institute 2017 (CLSI, 2017), with the aim of avoiding bacteremia or sepsis of in immunocompromised patients. Antimicrobial susceptibility testing of clinical isolates is also recommended by the CLSI guidelines (6). Additional studies of actions and medications that influence in the bacterial adherence may contribute to more effective drugs and new guidelines.

In the present article, the case of an L. aquatica infection (before Corynebacterium aquaticum infection) isolated from a pediatric patient with a Wilms tumor who was kept on a catheter long term is described. This bacteria is a bacilli gram positive, and has a natural affinity for moist surfaces and or water-based solutions and can infect animals and plants, including human beings (7). This paper should be of interest to a broad readership including those interested in demonstrated that treatment of these infections may contribute to significant changes in clinical and epidemiological features of corynebacterial infections in nosocomial environments. 


\section{Case report}

This study received Institutional Review Board approval from the National Cancer Institute (INCA/HCI, Rio de Janeiro, Brazil; approval no. CAAE- 0121.0.007.000-11).

The present report describes the of a 4-year-old boy, white, with hematuria, dysuria and abdominal pain. He had a palpable abdominal mass, and this underwent physical examination. In the contrast-enhanced computerized tomography, the right kidney was normal whereas the left kidney presented a mass $(7.4 \times 6.2 \mathrm{~cm})$ in the upper third of the right kidney. Following the New Wilms Tumor Study (NWTS) protocol, surgical intervention was recommended $(8,9)$. Surgical enquiry showed the presence of a large tumor in the left kidney with renal homolateral vein thrombosis. Nephro-adrenalectomy was carried out with retroperitoneal lymphadenectomy and thrombectomy. Subsequently, the tumor was classified as stage EIII (referring to a Wilms tumor that has most likely not been removed completely, for example, in the case where a disease has spread to lymph nodes). On 9th of February 2002, under the NWTS EE4A protocol, the patient was treated with doxorubicin and vincristine. During the first cycle of treatment, the patient presented with pneumopathy, and thus, the treatment was changed to ciprofloxacin. Subsequently, other infections occurred, including pultaceous tonsillitis (treated with amoxicillin) and urinary infection on the 12th of February 2003 (treated with Bactrim until 24th of April 2003.

During ambulatorial follow-up on the 10th of January 2004 due to chest pain, he was diagnosed with a recurring thoracic tumor, and was thus prescribed chemotherapy under NWTS for EV protocol with doxorubicin, vincristine and cyclophosphamide. Radiotherapy of the thorax and thoracic spine column was recommended and was performed between 8th of January 2004 until 2nd of February 2004. A Venous Catheter of Long Permanency (CVC LP SI) was inserted on the 29th of January 2004, in the humeral vein under institutional protocol. On 17th of February 2004, the patient developed an Enterobacter cloacae urinary infection in the right kidney, which was treated with ciprofloxacin antibiotic for 10 days $(15 \mathrm{mg} / \mathrm{kg} / \mathrm{day})$. During the chemotherapy cycle, he exhibited fever. On 23rd of February 2004, he had a venous prothesis, and was treated with cefalexin. As hemocultures were negative, the catheter was maintained. On the 15th of April 2004, he was diagnosed with pancytopenia and right pneumopathy, and was thus treated with cefepime; on the 21st of April 2004, he was considered neutropenic and he presented with herpes zoster in the perineal area, thus fluconazole was used for 10 days. On 21 May 2004, within 5 months of CVC, he had fungemia. Fluconazole was given, and the CVCLP SI (LSM) was removed. A peripheric intravenous central catheter was inserted on 3rd of June 2004. However, after 7 days, he had local hyperemia and pain, and thus it was removed. On 24th of June 2004, a new CVC LP SI was inserted in the RSM, and chemotherapy treatment was maintained. After 8 days, the patient presented with a new fever with neutropenia, and thus ceftriaxone was started. During doppler ultrasound examination, a thrombus in the right atrium and cultures from the insertion ostium of the CVC was identified. On 9th of July 2004, a non-solid mass in the lung associated with disseminated intravascular coagulation occurred. Culturing and analysis confirmed the growth of L. aquatica associated a member of the Staphylococcus spp. taxa. Thus, the catheter was removed, and the patient was administered amikacin and vancomycin in the intensive therapy. Coagulation examinations showed: Antithrombin III-112\%, anticardiolipin (+), C reactive protein $50.2 \mathrm{mg} / \mathrm{dl}$ and lupus anticoagulant (-); thus, anti-coagulation therapy was started on enoxaparin $20 \mathrm{mg}$ /day for anti-phospholipidic syndrome (condition of oncogenic origin, and due to a family history of hereditary hemostasis). A third CVC LP catheter was inserted on the 5th of August 2004, which was removed on 24th of March 2005, after sufficient control of hemostasis was achieved. Patient underwent ambulatorial follow-up on 23rd of July 2014, were co-axial tomography showed a relapse of thoracic disease on the right side, and a venous chemotherapy cycle was started under the Vincristine, Irinotecan, Temozolomide and Bevacizumab protocol with a new CVC LP inserted in the right subclavian on the 18th of July 2014, and a reduction of the mass was achieved. He remained under thoracotomy until 12th of January 2015 (10). During the post-surgery period, he presented with the following complications: Suture dehiscence in the surgical wound, and infection by Methicillin-resistant Staphylococcus aureus and Acinetobacter spp. pathogens. He was treated with linezolid and ciprofloxacin. After bandaging and surgical interventions, the wound completely healed. Chemotherapy was ended on 16th of November 2015. Subsequently, the patient came back for ambulatorial follow-up, and there was no evidence of disease and/or infection.

\section{Discussion}

In the last two decades, Non-diphtheria Corynebacterium species have been increasingly recognized as MDR pathogens that also infect immunocompromised patients, which may be caused by inappropriate outpatient prescription of antibiotics $(3,5,11-13)$. There are reports of isolation of coryneform bacteria causing nosocomial infections, such as endocarditis, and orthopedic bone and joint infections (14). Isolates of coryneform gram-positive rods are often difficult to identify for diagnostic laboratories (15).

There are only a few reports describing an L. aquatica infection in an individual under long-term catheter use (14-19). An integrative review was performed based on the following guiding question: How common is an L. aquatic infection? And what is best antibiotic approach to treat it, primarily in immunocompromised patients?

A literature review was performed by searching SCOPUS and MEDLINE, using the key words in medical subject heading (MeSH): 'Leifsonia aquatica', 'catheter', 'neoplasms'. Key words were combined using the Boolean operator 'AND'.

The inclusion criteria were as follow: Original articles, letters to the editor and bibliographic reviews published in English. No timeframe was set in this research. The evaluated aspects were: Documents by type, documents by country/territory, documents by affiliation, documents by author, periodic research and publication by year. Articles that were considered other aspects of L. aquatica infections related were excluded from this review. 


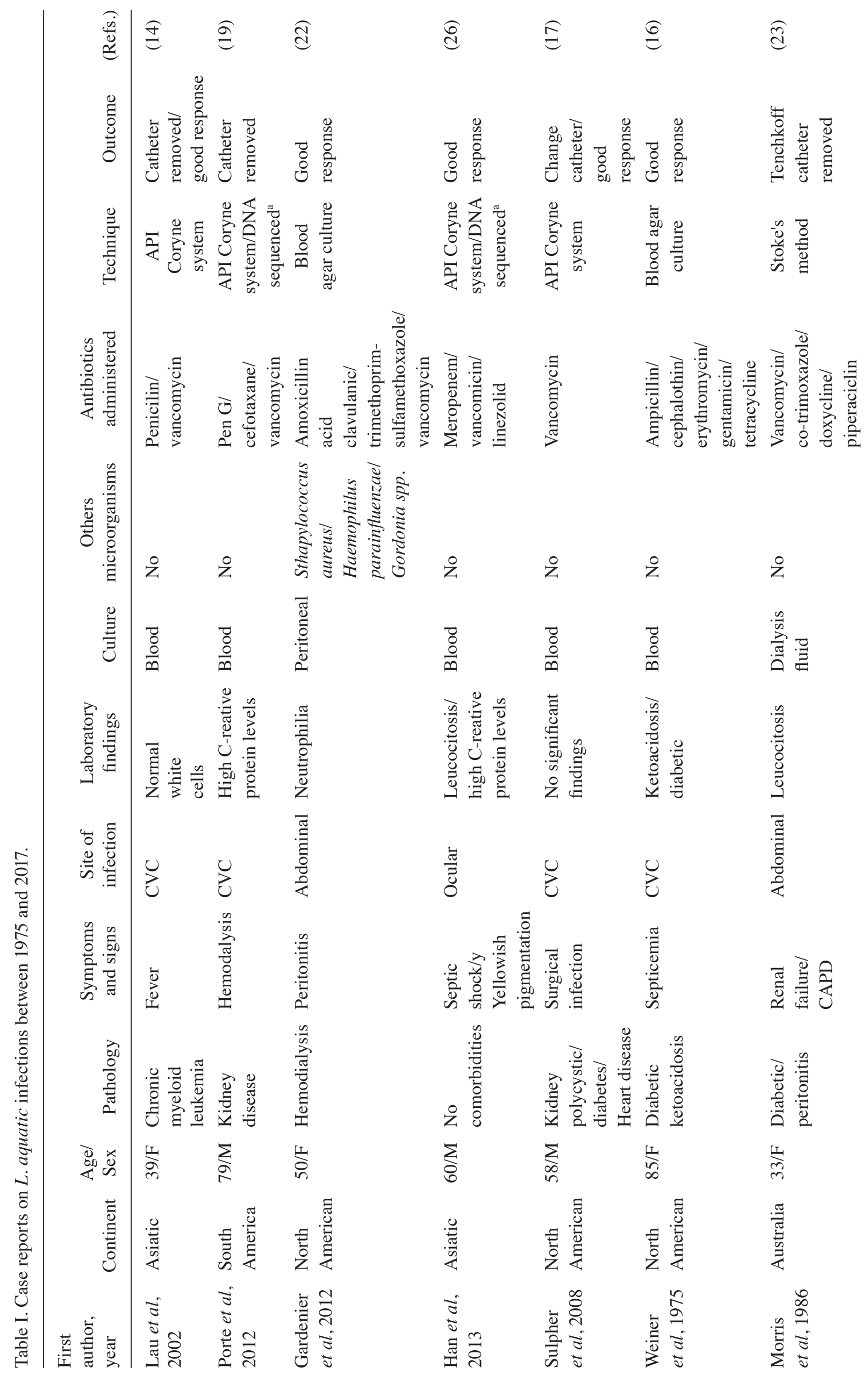




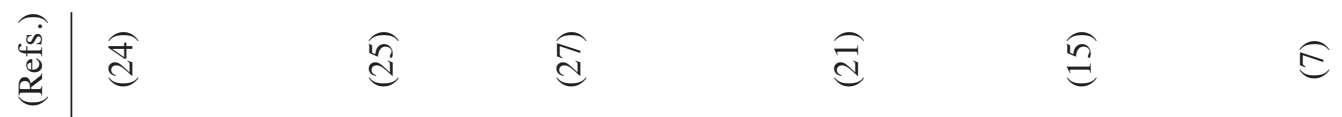

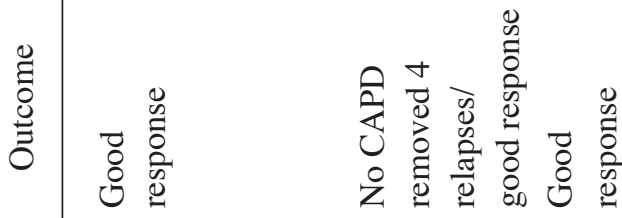

节

预融

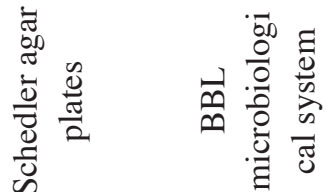

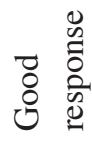

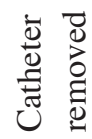

焉

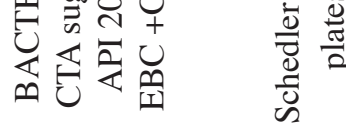

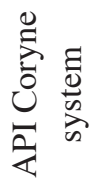

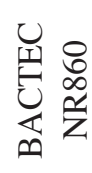

苍毒

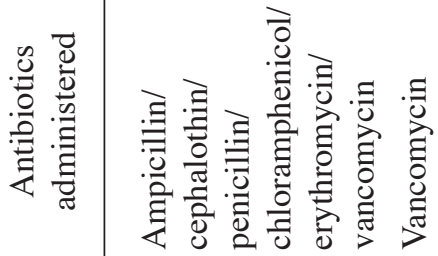

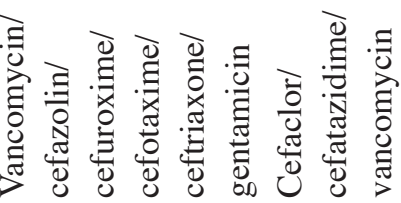

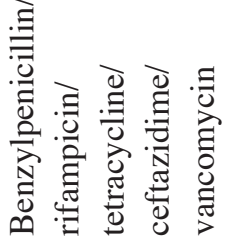

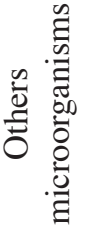

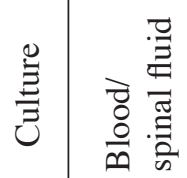

ż

ฉ

$\gtrless$

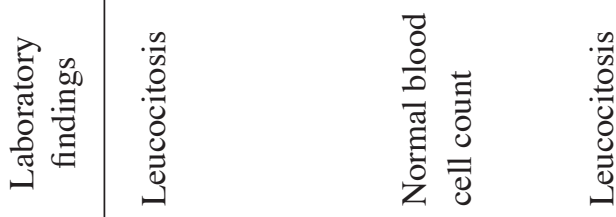

站 密

$\frac{\square}{\circ}$

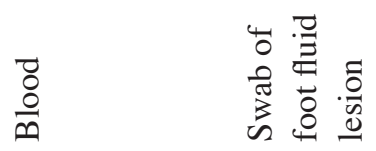

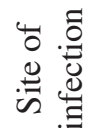

言

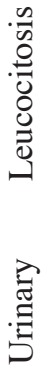

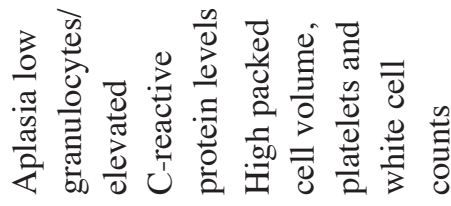

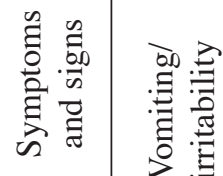

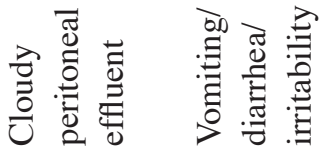

之龸

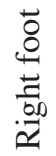

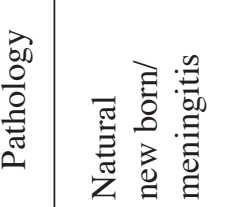

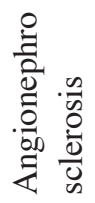

总

紫

离

总

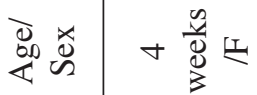

i

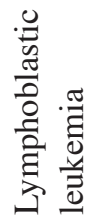

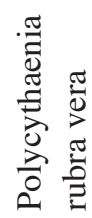

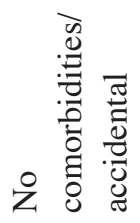

泀

壱离

$\infty$ 商 $\Sigma$

$\sum_{m}$

竞

$\underset{\text { 住 }}{\sum}$

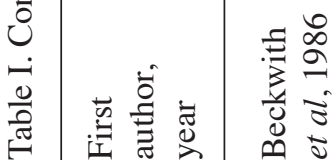

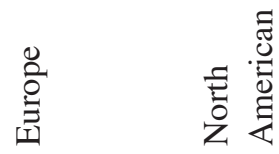

芒

丞

产

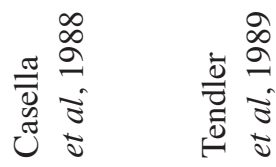

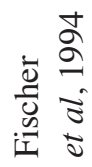

高离

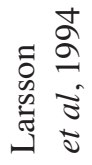




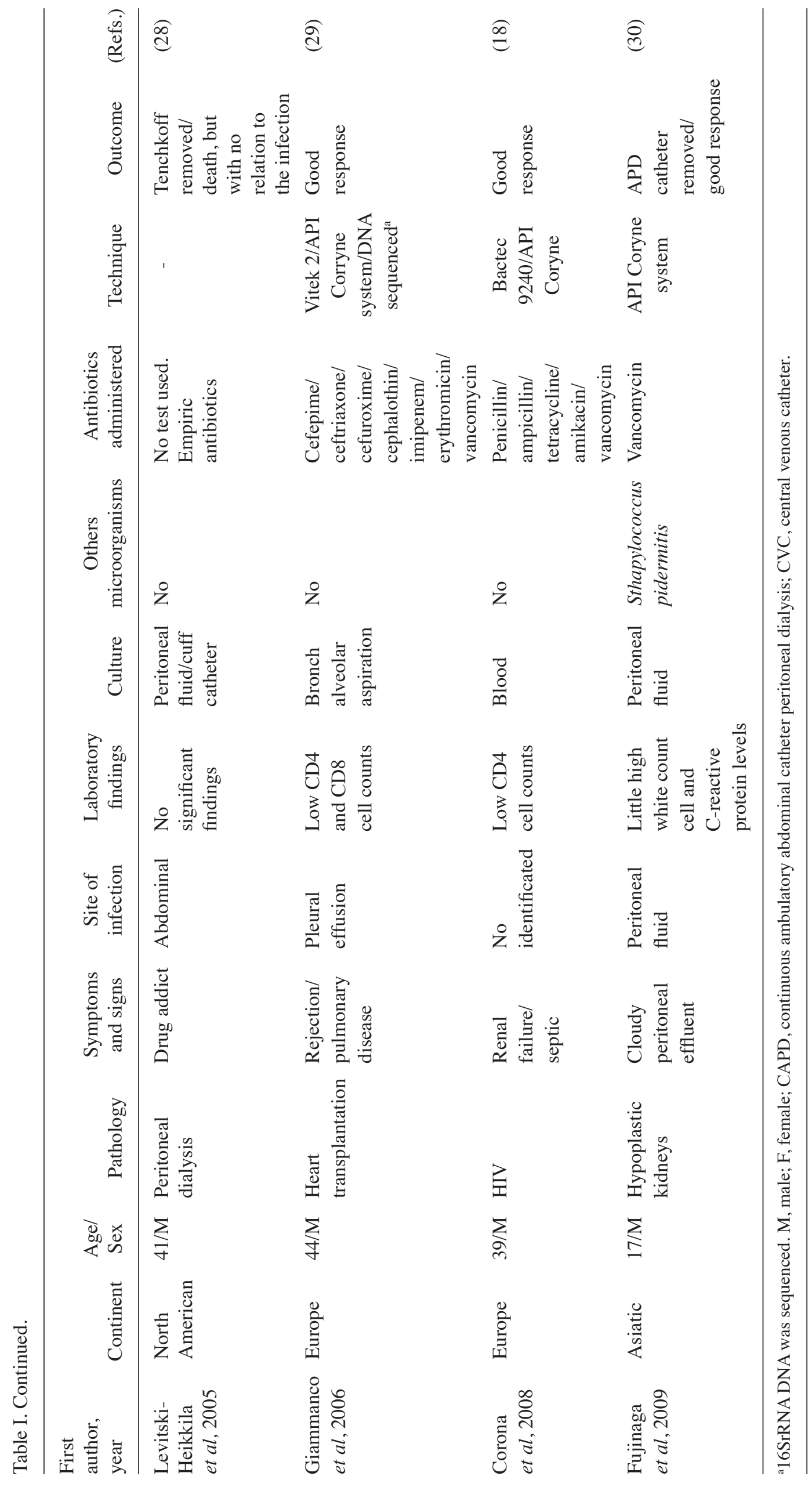


Information on the cause of the case that was defined as being of importance for discussion in this integrative review. The description in the title and abstracts were assessed in the filtered articles obtained. Evaluation and critical analysis of the articles involved classifying them by case reports in immunocompromised patients.

A review of the literature revealed a case report involving dialysis patients with documented L. aquatica bacteremia (19) Searching the two databases, SCOPUS and MEDLINE. In SCOPUS, 15 articles were retrieved; and 3 were discarded as they were deemed irrelevant based on either the title or the abstract. Thus 12 articles were obtained. In MEDLINE, 17 articles were selected but articles were the written language was not English were discarded, and 1 article was a duplicate between the two databases. The case reports selected after filtering out irrelevant articles are shown in Table I. The source basis, signals and symptoms, the laboratory results, comorbidities associated, equipment used to identify L.aquatic infection, the susceptible antibiotic tests used in each related case report and the outcome of each case patient is described. This review of the literature did not reveal any other case report describing the case of an L.aquatica infection in a patient with cancer or a pediatric patient.

In the present report, the rare case of an L. aquatica infection isolated from a pediatric patient with a Wilms tumor is described. To the best of our knowledge, this is the only report of an L. aquatica infection in Brazil associated with long term catheter use. This report highlights the possibility of a rare case of L. aquatica infection isolated during chemotherapy treatment for Wilms Tumor, and the clinical aspects of this patient, and the subsequent developments for improving treatment were analyzed.

A study by Adderson et al describes some cases of Coryneform bacterial infections in a pediatric oncological setting. They proposed that the incidence and presentation of these infections differed in children and adults (20).

In the present study, L. aquatica was responsible for central venous catheter related infection in the pediatric patient with solid malignant tumor. Most reports of corynebacterial infections describe single patients, and thus the characteristics of these infections are poorly defined (17,21-26). Certain articles highlight the pathogenicity of this bacterium as the primary causative agent of bacteremia in patients on dialysis and is not considered a simple episode of opportunistic infection (27-30).

Vancomycin has been considered the treatment of choice for management of corynebacterial infections $(11,31)$. Indeed, the majority of reports from Europe show C. Jeikeium as susceptible to vancomycin, but resistant to other antibiotics $(5,32)$. The biofilm on catheter surfaces formed by this agent complicates eradication of this bacteria (33). Additional complications, such as longer hospitalization periods, use of other catheters or a non-competent patient increase the necessity of catheter removal. Thus, introducing vancomycin for management of these infections may be a sensible approach to reduce the risk of worsening of infections. Vancomycin remains the better option as empiric treatment of catheter Corynebacterium infection $(34,35)$. This approach reduces the risk of making an assumption of the nature of the pathogen based on 'common sense', which could increase the risk to the patient. Instead, correct identification and treatment can prevent further complications.

In Conclusion, infections by Corynebacterium species are increasingly being recognized as causative agents of bloodstream and venous catheter-related infections in immunocompromised patients. With the development and advancement of novel technologies and improvement in diagnostic approaches, and the possibility of using new medications guided by accurate clinical laboratory identification with suitable sensitivity, non-Corynebacterium may be implicated in an increasing number of deaths in immunocompromised patients. This literature review highlights the increasing importance of the potential for infections and the problems they can cause in patients using catheters.

\section{Acknowledgements}

Not applicable.

\section{Funding}

This study was fully supported by the Research Support Foundation of Rio de Janeiro (FAPERJ), National Council for Cientific and Technological Development (CNPq) and Ministry of Health-Science and Technology-National Cancer Institute; INCA/MS.

\section{Availability of data and materials}

The datasets used and/or analyzed during the present study are available from the corresponding author on reasonable request.

\section{Authors' contributions}

ALMG designed the study, analyzed and interpreted the data, and assisted in writing the manuscript. RVDC performed the experiments, interpreted the results, drafted the manuscript, performed the surgical procedure and logged the patient's data/results, and performed the analysis. MFG was responsible for patient treatment and welfare. FFL and LSDSSA performed the experiments, interpreted the results and drafted the manuscript. CSS and RHJ assisted in writing the manuscript, and performed the microbiological data analysis and interpretation. All authors have read and approved the final manuscript. ALMG, CSS and RVDC confirm the authenticity of all the raw data.

\section{Ethics approval and consent to participate}

This study received Institutional Review Board approval from the National Cancer Institute (INCA/HCI, Rio de Janeiro, Brazil; approval no. CAAE- 0121.0.007.000-11).

\section{Patient consent for publication}

Not applicable.

\section{Competing interests}

The authors declare that they have no competing interests. 


\section{References}

1. Leal SM Jr, Jones M and Gilligan PH: Clinical significance of commensal gram-positive rods routinely isolated from patient samples. J Clin Microbiol 54: 2928-2936, 2016.

2. Bernard KA, Pacheco AL, Burdz T and Wiebe D: Increase in detection of Corynebacterium diphtheriae in Canada: 2006-2019. Can Commun Dis Rep 45: 296-301, 2019

3. Camello TCF, Mattos-Guaraldi AL, Formiga LCD and Marques EA: Non-diphtherial Corynebacterium species isolated from clinical specimens of patients in a University Hospital, Rio de Janeiro, Brazil. Brazilian J Microbiol 34: 39-44, 2003.

4. Barberis C, Almuzara M, Join-Lambert O, Ramírez MS Famiglietti A and Vay C: Comparison of the Bruker MALDI-TOF mass spectrometry system and conventional phenotypic methods for identification of Gram-positive rods. PLoS One 9: e106303, 2014.

5. Martins CAS, Faria LMD, Souza MC, Camello TCF, Velasco E, Hirata R, Jr, Thuler LCS and Mattos-Guaraldi AL: Microbiological and host features associated with corynebacteriosis in cancer patients: A five-year study. Mem Inst Oswaldo Cruz 104: 905-913, 2009.

6. CLSI: Performance Standards for Antimicrobial Susceptibility Testing. 474 M100-S27. Clinical and Laboratory Standards Institute, Wayne, PA, 2017.

7. Larsson P, Lundin O and Falsen E: 'Corynebacterium aquaticum' wound infection after high-pressure water injection into the foot. Scand J Infect Dis 28: 635-636, 1996

8. Bhatnagar S: Management of Wilms' tumor: NWTS vs. SIOP. J Indian Assoc Pediatr Surg 14: 6-14, 2009.

9. Kieran K and Ehrlich PF: Current surgical standards of care in Wilms tumor. Urol Oncol 34: 13-23, 2016.

10. Venkatramani R, Malogolowkin MH and Mascarenhas L: Treatment of multiply relapsed wilms tumor with vincristine, irinotecan, temozolomide and bevacizumab. Pediatr Blood Cancer 61: 756-759, 2014.

11. Carvalho RV, Lima FFDS, Santos CSD, Souza MC, Silva RSD and Mattos-Guaraldi AL: Central venous catheter-related infections caused by Corynebacterium amycolatum and other multiresistant non-diphtherial corynebacteria in paediatric oncology patients. Braz J Infect Dis 22: 347-351, 2018.

12. Milani RV, Wilt JK, Entwisle J, Hand J, Cazabon P and Bohan JG: Reducing inappropriate outpatient antibiotic prescribing: Normative comparison using unblinded provider reports. BMJ Open Qual 8: e000351, 2019.

13. Imai M, Kimura Y, Tanno D, Saito K, Honda M, Takano Y, Ohashi K, Toyokawa M, Ohana N, Yamadera Y and Shimura H: Validation of MALDI-TOF MS devices in reanalysis of unidentified pathogenic bacteria detected in blood cultures. Fukushima J Med Sci 66: 103-112, 2020

14. Lau SK, Woo PC, Woo GK and Yuen KY: Catheter-related Microbacterium bacteremia identified by $16 \mathrm{~S}$ rRNA gene sequencing. J Clin Microbiol 40: 2681-2685, 2002.

15. Moore $\mathrm{C}$ and Norton R: Corynebacterium aquaticum septicemia in a neutropenic patient. J Clin Pathol 48: 971-972, 1995.

16. Weiner M and Werthamer S: Corynebacterium aquaticum Septicemia: Characterization of the Microorganisms. Am J Clin Pathol 64: 378-381, 1975

17. Sulpher J, Desjardins M and Lee BC: Central venous catheter-associated Leifsonia aquatica bacteremia in a hemodialysis-dependent patient. Diagn Microbiol Infect Dis 61: 64-66, 2008.

18. Corona A, Castelli A, Ballone E, Rech R, Colombo R, Ferraris S, Della Porta V and Raimondi F: A case of septic shock secondary to Corynebacterium aquaticum bacteremia occurring in an HIV-infected patient attending a promiscuous thermal SPA Minerva Anestesiol 74: 213-215, 2008.

19. Porte L, Soto A, Andrighetti D, Dabanch J, Braun S, Saldivia A Flores JC, Wozniak A, García P and Weitzel T: Catheter-associated bloodstream infection caused by Leifsonia aquatica in a haemodialysis patient: A case report. J Med Microbiol 61: 868-873, 2012 .
20. Adderson EE, Boudreaux JW and Hayden RT: Infections caused by coryneform bacteria in Pediatric Oncology Patients. Pediatr Infect Dis J 27: 136-141. 2008.

21. Fischer RA, Peters G, Gehrmann $J$ and Jürgens $H$ : Corynebacterium aquaticum septicemia with acute lymphoblastic leukemia. Pediatr Infect Dis 13: 836-837, 1994.

22. Gardenier JC, Sawyer RG, Sifri CD, Brayman K, Wispelway B and Bonatti H: Peritonitis caused by Haemophilus parainfluenzae, Leifsonia aquatica, and Gordonia spp. in a patient undergoing continuous ambulatory peritoneal dialysis. Surg Infect (Larchmt) 13: 409-412, 2012.

23. Morris AJ, Henderson GK, Bremner DA and Collins JF: Relapsing peritonitis in a patient undergoing continuous ambulatory peritoneal dialysis due to Corynebacterium aquaticum. J Infect 13: 151-156, 1986.

24. Beckwith DG, Jahre JA and Haggerty S: Isolation of Corynebacterium aquaticum from spinal fluid of an infant with meningitis. J Clin Microbiol 23: 375-376, 1986.

25. Casella P, Bosoni MA and Tommasi A: Recurrent Corynebacterium aquaticum peritonitis in a patient undergoing continuous ambulatory peritoneal dialysis. Clin Microbiol Newsl 10: 62-63, 1988.

26. Han L, Lei JE, Wang X, Guo LT, Kang QY, He L and Xu JR Septicemia caused by Leifsonia aquatica in a healthy patient after retinal reattachment surgery. J Clin Microbiol 51: 3886-3888, 2013.

27. Tendler $\mathrm{C}$ and Bottone EJ: Corynebacterium aquaticum urinary tract infection in a neonate and concepts regarding the role of the organism as a neonatal pathogen. Clin Microbiol 27: 343-345, 1989.

28. Levitski-Heikkila TV and Ullian ME: Peritonitis with multiple rare environmental bacteria in a patient receiving long-term peritoneal dialysis. Am J Kidney Dis 46: e119-e124, 2005.

29. Giammanco GM, Pignato S, Grimont PA, Grimont F, Santangelo C, Leonardi G, Giuffrida A, Legname V and Giamanco G: Interstitial pulmonary inflammation due to Microbacterium $s p$. after heart transplantation. J Med Microbiol 55: 335-339, 2006

30. Fujinaga S, Ohtomo Y, Hirano D, Nishizaki N, Someya T, Ohtsuke Y, Kaneko K and Shimizu T: Relapsing peritonitis with Corynebacterium aquaticum in a boy receiving automated peritoneal dialysis. Pediatr Nephrol 24: 1253-1254, 2009.

31. Yang K, Kruse RL, Lin WV and Musher DM: Corynebacteria as a cause of pulmonary infection: A case series and literature review. Pneumonia (Nathan) 5: 10, 2018

32. Riebel W, Frantz N, Adelstein D and Spagnuolo PJ: Corynebacterium JK: A cause of nosocomial device-related infection. Rev Infect Dis 8: 42-49, 1986.

33. Darouiche RO, Dhir A, Miller AJ, Landon GC, Raad II and Musher DM: Vancomycin penetration into biofilm covering infected prostheses and effect on bacteria. J Infect Dis 170: 720-723, 1994.

34. Ghide S, Jiang Y, Hachem R, Chaftari AM and Raad I: Catheter related Corynebacterium bacterium bacteremia: Should the catheter be removed and vancomycin administered? Euro J Clin Microbiol Infect Dis 29: 153-156, 2010.

35. Yanai M, Ogasawasa M, Hayashi Y, Suzuki K, Takahashi H and Satomura A: Retrospective evoluation of the clinical characteristics associated with Corynebacterium species bacteremia. Braz J Infect Dis 22: 24-29, 2018.

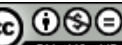

This work is licensed under a Creative Commons Attribution-NonCommercial-NoDerivatives 4.0 International (CC BY-NC-ND 4.0) License. 